\title{
The intrinsic dispersion in the Faber-Jackson relation for early-type galaxies as function of the mass and redshift
}

\author{
A. Nigoche-Netro ${ }^{1}$, J. A. L. Aguerri²,3 ${ }^{2}$ P. Lagos $^{4}$, A. Ruelas-Mayorga ${ }^{5}$, L. J. Sánchez ${ }^{5}$, \\ C. Muñoz-Tuñón ${ }^{2,3}$, and A. Machado ${ }^{5}$ \\ ${ }^{1}$ Instituto de Astrofísica de Andalucía (IAA), Glorieta de la Astronomía s/n, 18008 Granada, Spain \\ e-mail: nigoche@iaa.es \\ 2 Instituto de Astrofísica de Canarias (IAC), vía Láctea s/n, 38200 La Laguna, Spain \\ e-mail: jalfonso \\ 3 Departamento de Astrofísica, Universidad de La Laguna, 38206 La Laguna, Spain \\ ${ }^{4}$ Centro de Astrofisica da Universidade do Porto, Rua das Estrelas, 4150-762 Porto, Portugal \\ e-mail: plagos@astro.up.pt \\ 5 Instituto de Astronomía, Universidad Nacional Autónoma de México, Apartado Postal 70-264, Cd Universitaria 04510 México \\ D.F., México \\ e-mail: [rarm; leonardo;machado] @astroscu.unam.mx
}

Received 20 December 2010 / Accepted 19 July 2011

\section{ABSTRACT}

\begin{abstract}
Context. It has been reported that the intrinsic dispersion at constant magnitude in the structural relations of early-type galaxies is a useful tool to study the universality of these structural relations, that is to say, to study whether the structural relations depend on luminosity, wavelength, redshift, and/or environment.

Aims. We study the intrinsic dispersion at approximately constant magnitude in the Faber-Jackson relation as a function of luminosity, mass, and redshift.

Methods. We use a sample of approximately 90000 early-type galaxies from the Sloan Digital Sky Survey (SDSS-DR7) spanning a magnitude range of 7 mag in both $g$ and $r$ filters. We calculate the intrinsic dispersion in the Faber-Jackson relation at approximately constant magnitude and compare this at different luminosities, masses, and redshifts.

Results. The main results are the following: i) the intrinsic dispersion in the Faber-Jackson relation depends on the luminosity, mass, and redshift; ii) the distribution for brighter and more massive galaxies has smaller intrinsic dispersion than that for fainter and less massive galaxies; iii) the distribution of bright and massive galaxies at higher redshift has smaller intrinsic dispersion than similar galaxies at low redshift.

Conclusions. Comparisons of the results found in this work with recent studies from the literature make us conclude that the intrinsic dispersion in the Faber-Jackson relation could depend on the history of galaxies, in other words, the intrinsic dispersion could depend on the number and nature of transformation events that have affected the galaxies during their life times, such as collapse, accretion, interaction, and merging.
\end{abstract}

Key words. galaxies: distances and redshifts - galaxies: elliptical and lenticular, CD - galaxies: fundamental parameters

\section{Introduction}

The scaling relations of early-type galaxies (ETGs) are mathematical relations involving the structural parameters of galaxies such as: effective radius $\left(r_{\mathrm{e}}\right)$, mean effective surface brightness inside $r_{\mathrm{e}}\left(\langle\mu\rangle_{\mathrm{e}}\right)$, central velocity dispersion $\left(\sigma_{0}\right)$, and total absolute magnitude $(M)$. Among these structural relations, we have the Kormendy relation (KR; Kormendy 1977), the Faber-Jackson relation (FJR; Faber \& Jackson 1976), and the fundamental plane (FP; Djorgovsky \& Davis 1987; Dressler et al. 1987), which are very useful tools for understanding the processes of formation and evolution of galaxies. There have been many efforts to investigate the properties of ETGs using the structural relations. The vast majority of these works have studied the coefficients of the structural relations at different wavelengths, environments, redshifts, and luminosities and the results that they have found are very heterogeneous and discrepant. For example, several studies have found that the coefficients of the structural relations remain stable when considering different wavelengths (Bender et al. 1992; Bender et al. 1998; Bernardi et al. 2003b; Bernardi et al. 2003c; La Barbera et al. 2005; La Barbera et al. 2008). However, other studies show that the structural relations depend on wavelength (Jørgensen et al. 1996; Hudson et al. 1997; Pahre et al. 1998; Scodeggio et al. 1998; Jun \& Im 2008). Several studies have shown that the structural relations and/or the structural parameters of galaxies are affected by the environment (Bender et al. 1992; Bender et al. 1998; Trujillo et al. 2001; Trujillo et al. 2002; Bernardi et al. 2003b; Aguerri et al. 2004; Gutiérrez et al. 2004; Denicoló et al. 2005; Jørgensen et al. 2005), although other studies have found the opposite conclusion (de la Rosa et al. 2001; Treu et al. 2001; Evstigneeva et al. 2002; Gonzáles-García \& van Albada 2003; Reda et al. 2004; Reda et al. 2005; Nigoche-Netro et al. 2007). When structural relations of galaxy samples are studied at different redshifts, several studies indicate that only the zero point of these relations depends on the redshift (Barger et al. 1998; Ziegler et al. 1999; La Barbera et al. 2003; Barr et al. 2006). However, other authors find that there is, not only a dependence 
of the zero point on redshift but that the slopes of the structural relations are steeper for higher redshift galaxies than for galaxies in the local Universe (Treu et al. 2005; Jørgensen et al. 2006; Fritz et al. 2009). Finally, when considering different luminosity, some authors find that dwarf and bright ellipticals follow structural relations with different coefficients (Kormendy 1985; Hamabe \& Kormendy 1987; Bender et al. 1992; Caon et al. 1993; Aguerri \& González-García 2009; Desroches et al. 2007). Nigoche-Netro (2007) and Nigoche-Netro et al. (2008, 2009, 2010) find that the distribution of galaxies in the space of parameters defining the structural relations depends on the luminosity and that the coefficients of the structural relations depend on the width of the magnitude range $(\Delta M)$ within which the galaxies are contained. They also find that when the width of the magnitude range diminishes, the differences in the slope of the structural relations (for intervals of the same width and different luminosity) become small and when it has approximately constant magnitude the differences are negligible. This effect is present in all samples of galaxies studied, independently of their degree of intrinsic difference, and has been referred to as a "geometrical effect".

In contrast, the intrinsic dispersion has been less well-studied in the astronomical literature than the values of the coefficients of the structural relations. Some papers have found that this dispersion depends on both luminosity (Bender et al. 1992; Jørgensen et al. 1996; Hyde \& Bernardi 2009) and the environment (Bernardi et al. 2003b; Denicoló et al. 2005). The behaviour of the intrinsic dispersions in the FJR and the KR have not been studied thoroughly because a third variable is believed to cause most of the intrinsic dispersion in both these relations. The intrinsic dispersion in the FP has been barely studied because, it has been assumed to be small ( $\sim 0.1$ dex, Kjærgaard et al. 1993; Jørgensen et al. 1996; Kelson et al. 1997; Jørgensen et al. 1999; Blakeslee et al. 2002; Bernardi et al. 2003c; Reda et al. 2005; Jørgensen et al. 2006). However, some studies have found that the intrinsic dispersion is far from small ( $\sim 0.3 \mathrm{dex}$, Bender et al. 1992; La Barbera et al. 2003; Nigoche-Netro et al. 2009). This relatively large dispersion implies that the galaxy distribution in space that defines the structural relations follows a surface whose thickness is determined by this dispersion.

Nigoche-Netro (2007) and Nigoche-Netro et al. (2008, 2009, 2010) demonstrated that the intrinsic dispersion is also affected by the geometrical effect. However, Nigoche-Netro et al. (2010) found that differences between the intrinsic dispersions of different samples of galaxies do not disappear when the magnitude range tends to smaller values. They discovered that the true value of the intrinsic dispersion can be measured when $\Delta M=0$. The intrinsic dispersion in this extreme case would be defined as the standard deviation in the distribution of the points at constant magnitude. Hence, Nigoche-Netro et al. (2010) propose that a valuable way of obtaining the physical properties of a sample of galaxies is to find its intrinsic dispersion at each magnitude value and compare this dispersion with those for different luminosities, wavelengths, redshifts, or environments. In this paper, we carry out such a study of the behaviour of the intrinsic dispersion in the FJR as a function of luminosity, mass, and redshift for a sample of ETGs selected from the SDSS-DR7 archive.

In Sect. 2, we present the galaxy sample used to study the intrinsic dispersion in the FJR, the calculation of the intrinsic dispersion, and the analysis of the behaviour of the intrinsic dispersion as a function of the luminosity, mass, and redshift. In Sect. 3, we present a discussion of the most important results of this paper. Finally in Sect. 4 we present our conclusions.

\section{The intrinsic dispersion in the structural relations for a sample of ETGs}

\subsection{The sample of ETGs}

We use a sample of ETGs from the Seventh Data Release of the SDSS (York et al. 2000; Abazajian et al. 2009) in the $g$ and $r$ filters. This sample contains approximately 90000 galaxies in each filter, distributed across a redshift interval $0.01<z<$ 0.35 and within a magnitude range $\Delta M \sim 7 \mathrm{mag}$. The sample selection procedure was based on those of Bernardi et al. (2003a) and Hyde \& Bernardi (2009) (see Nigoche-Netro et al. 2010). Hereafter we refer to it as the total SDSS sample. Given that the total sample spans a relatively ample redshift range, it is affected by the Malmquist bias. To avoid this bias, we use a volumelimited sample of approximately 17000 ETGs with $0.04 \leq z \leq$ 0.08 in the $g$ and $r$ filters. This subsample covers a magnitude range $\langle\Delta M\rangle \sim 4.5 \mathrm{mag}\left(-18.5 \geq M_{g}>-23.0\right)$ in both filters and we refer to it as the homogeneous SDSS sample. This sample is approximately complete for $M_{g} \leq-20.0$.

\subsection{The structural relations of the ETGs and their intrinsic dispersions}

Nigoche-Netro (2007) and Nigoche-Netro et al. (2008, 2009, 2010) demonstrated that because of the geometrical effect that they discovered, the physical properties of galaxies cannot be reliably constrained by comparing the slopes of the structural relations for magnitude ranges of different widths or for magnitude ranges of the same width but of different luminosity, this is because, with the exception of the full magnitude interval, there is no ideal width at which comparisons should be made. Hence using the slopes of the structural relations to find intrinsic differences among samples of galaxies is a delicate matter, and the results can be inconclusive. This procedure should be supplemented by an alternative corroborative method. Nigoche-Netro et al. (2010) demonstrated that measuring the intrinsic dispersion of a scaling relation at constant magnitude is an appropriate method for obtaining physical information about a sample of galaxies. In the subsequent sections, we use this method to try to find differences among the structural properties of galaxies belonging to different samples.

\subsection{The intrinsic dispersion in the FJR as function of luminosity, mass, and redshift}

\subsubsection{The intrinsic dispersion in the FJR as a function of luminosity}

Some papers from the literature have studied the intrinsic dispersion in the structural relations as a function of luminosity (Bender et al. 1992; Jørgensen et al. 1996; Hyde \& Bernardi 2009; Nigoche-Netro et al. 2010). Those works revealed that the intrinsic dispersion for bright galaxies is smaller than that for faint galaxies. However, these works compared the intrinsic dispersion for wide magnitude ranges, hence these results are affected by the geometrical effect. An appropriate analysis requires the calculation of the intrinsic dispersion at constant magnitude. In Fig. 1, we show the behaviour of the intrinsic dispersion in the FJR $\left(\sigma_{\log \left(\sigma_{0}\right)}\right)$ in very narrow magnitude ranges for the homogeneous and total samples from the SDSS in the $g$ filter. This figure shows the data for the homogeneous sample (red diamonds), where it is clearly seen that in the regime $M_{g} \lesssim-20$ for which the sample is complete, the intrinsic dispersion changes 
A. Nigoche-Netro et al.: The dispersion in the FJR as function of the mass and redshift

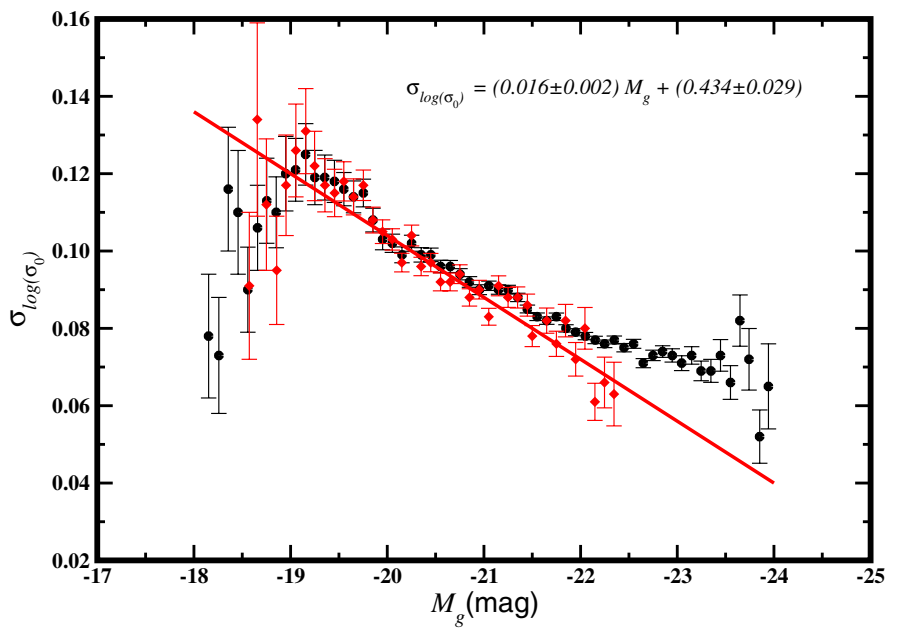

Fig. 1. Variation in the FJR intrinsic dispersion $\left(\sigma_{\log \left(\sigma_{0}\right)}\right)$ in 0.1 -mag wide intervals. Circles represent the total SDSS sample ( $g$ filter) and diamonds the homogeneous SDSS sample ( $g$ filter). The straight line represents a fit $\left(B C E S_{\mathrm{Bis}}\right)$ to the points in the homogeneous sample brighter than $M_{g} \sim-20$.

systematically as we consider brighter galaxies, and brighter galaxies have a smaller intrinsic dispersion than fainter galaxies. For the total SDSS sample (black dots), we observe the same behaviour.

We apply a non-parametric statistical test (run test) to check a randomness hypothesis for our data sequence (see NigocheNetro et al. 2009). More precisely, it can be used to test the hypothesis that the data of the intrinsic dispersion in the FJR are mutually independent. With this test, we find that there are reasons to affirm, with a $99 \%$ level of confidence, that there is an underlying trend for the intrinsic dispersion as a function of luminosity.

To characterise the behaviour of the intrinsic dispersion as a function of luminosity, we have fitted a straight line to those points in Fig. 1 that correspond to the homogeneous sample at $M_{g} \leq-20.0$. The resulting equation is:

$\sigma_{\log \left(\sigma_{0}\right)}=(0.016 \pm 0.002) M_{g}+(0.434 \pm 0.029)$.

The previous equation was obtained from a fit made with the bivariate correlated errors and intrinsic scatter bisector (BCES $S_{\text {Bis }}$ ) (Isobe et al. 1990; Akritas \& Bershady 1996) method. This method takes into consideration the errors in the variables, the error correlation, the data dispersion, and both variables as dependent variables. This method is used for all the fits in this paper.

\subsubsection{The intrinsic dispersion in the FJR as a function of mass}

We analyse the behaviour of the intrinsic dispersion in the FJR as a function of the mass (see also Shankar et al. 2011). We use two different methods to calculate the mass of galaxies. The first method requires the galaxies' luminosity and colour indices and the equation (see Bell et al. 2003)

$\mathbf{M}_{\mathbf{g}} \sim L_{g} 10^{a_{g}+b_{g}\left(M_{g}-M_{r}\right)}$,

where $\mathbf{M}_{\mathbf{g}}$ is the mass obtained from the luminosity in the $g$ filter $\left(L_{g}\right), M_{g}$ and $M_{r}$ are the magnitudes in the $g$ and $r$ filters, $a_{g}$ and $b_{q}$ are scale factors (see Table 7 from Bell et al. 2003). From

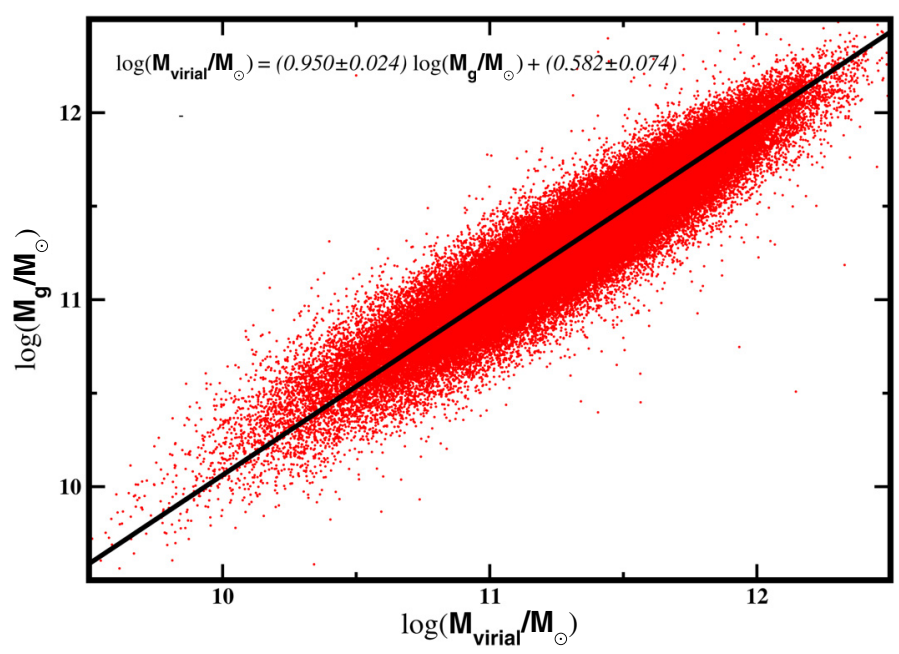

Fig. 2. Comparison of the mass obtained from the velocity dispersion and the mass obtained from the luminosity. The black line represents a fit $\left(B C E S_{\text {Bis }}\right)$ to the points in the total sample.

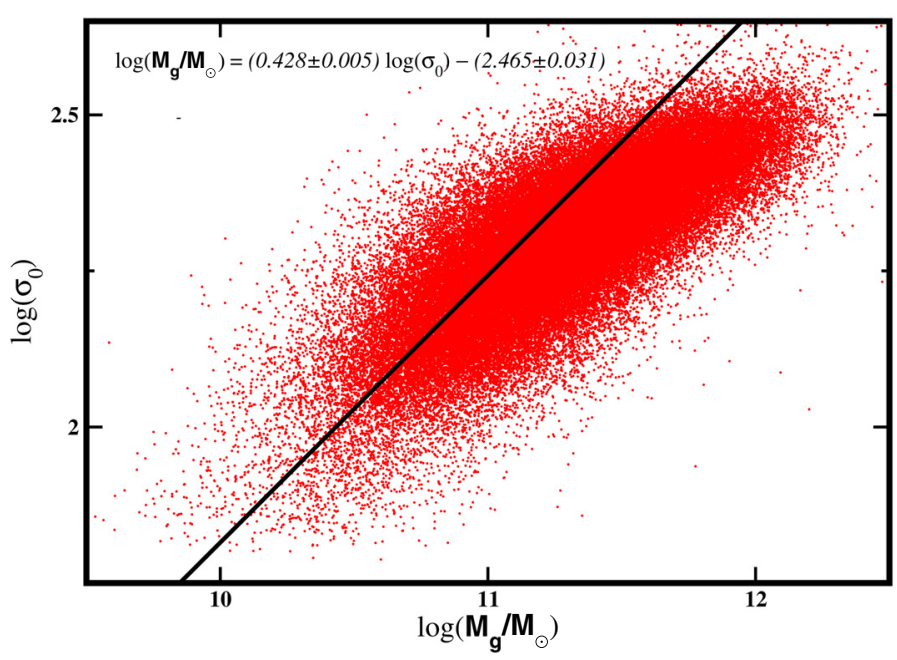

Fig. 3. Faber-Jackson relation using the mass obtained from the luminosity. The black line represents a fit $\left(B C E S_{\text {Bis }}\right)$ to the points in the total sample.

now on, the mass that we infer from the luminosity is called the stellar mass.

The second method requires knowledge of the velocity dispersion because it also assumes that the galaxies are in virial equilibrium and utilises the equation,

$\mathbf{M}_{\text {virial }} \sim \frac{5 r_{\mathrm{e}} \sigma_{0}^{2}}{G}$

where $\mathbf{M}_{\text {virial }}$ is the virial mass, $r_{\mathrm{e}}$ is the effective radius, $\sigma_{0}$ is the central velocity dispersion, and $G$ is the gravitational constant.

In Fig. 2, we present a comparison of the mass obtained using both methods. In Figs. 3 and 4, we see the relation between the mass and the velocity dispersion for the masses obtained with both methods. For a detailed discussion of Figs. 2-4, we refer to Sect. 2.3.3.

In Fig. 5, we show the behaviour of the virial mass as a function of redshift for galaxies contained in the SDSS total sample. Vertical lines represent the limits of the $0.04 \leq z \leq 0.08$ redshift interval where the homogeneous sample of the SDSS is contained. We note that within these limits there is a deficiency of galaxies for $\log \left(\mathbf{M}_{\text {virial }} / \mathbf{M}_{\odot}\right) \lesssim 10.5\left(\mathbf{M}_{\odot}\right.$ is the solar mass $)$, 


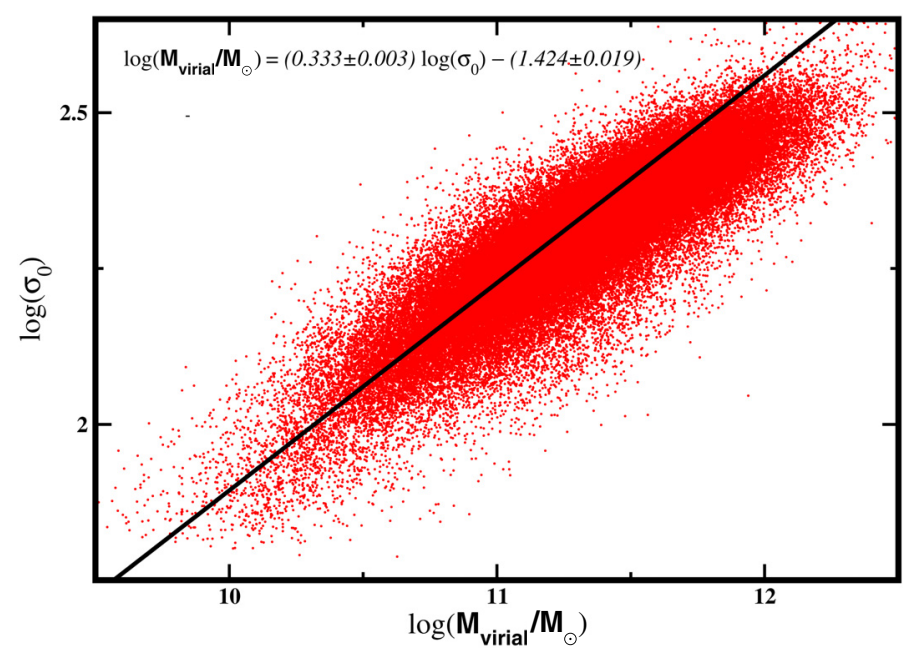

Fig. 4. Faber-Jackson relation using the mass obtained from the velocity dispersion. The black line represents a fit $\left(B C E S_{\text {Bis }}\right)$ to the points in the total sample.

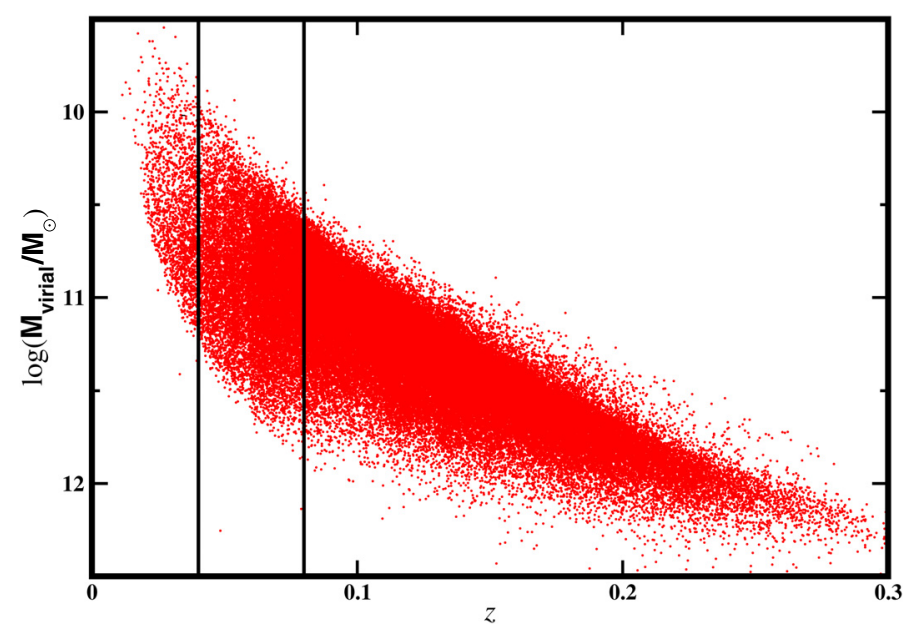

Fig. 5. Distribution of the mass as a function of redshift for the total SDSS sample. The vertical lines correspond to the $0.04 \leq z \leq 0.08$ redshift interval within which the homogeneous sample is contained. In this interval, we note that for $\log \left(\mathbf{M}_{\text {virial }} / \mathbf{M}_{\odot}\right) \lesssim 10.5$ there exists a deficiency of galaxies, so $\log \left(\mathbf{M}_{\text {virial }} / \mathbf{M}_{\odot}\right)=10.5$ could be considered to be the completeness limit of the homogeneous SDSS sample.

so we may affirm that $\log \left(\mathbf{M}_{\text {virial }} / \mathbf{M}_{\odot}\right)=10.5$ represents the approximate completeness limit of the homogeneous SDSS sample. On the other hand, the behaviour of the stellar mass as function of the redshift is similar to that of the virial mass, so that the approximate completeness limit for the homogeneous sample is, in this case, also $\log \left(\mathbf{M}_{\mathbf{g}} / \mathbf{M}_{\odot}\right)=10.5$.

In studying the intrinsic dispersion as a function of mass, we require calculation of the intrinsic dispersion at constant mass to avoid being affected by the geometrical effect. In Fig. 6, we show the behaviour of the intrinsic dispersion in the FJR in very narrow mass ranges $\left(0.1-\log \left(\mathbf{M}_{\mathbf{v i r i a l}} / \mathbf{M}_{\odot}\right)\right.$ wide intervals $)$ for the homogeneous and total samples from the SDSS. In this figure, we see that the values of the FJR intrinsic dispersion depend on the virial mass. However, this mass was obtained from Eq. (3), which involves both the effective radius as well as the velocity dispersion, that is to say, there is a correlation between the virial mass and velocity dispersion that might affect the intrinsic dispersion estimate (see Sect. 2.3.3 for more details). To avoid this possible bias, it is necessary to use the stellar mass. In Fig. 7,

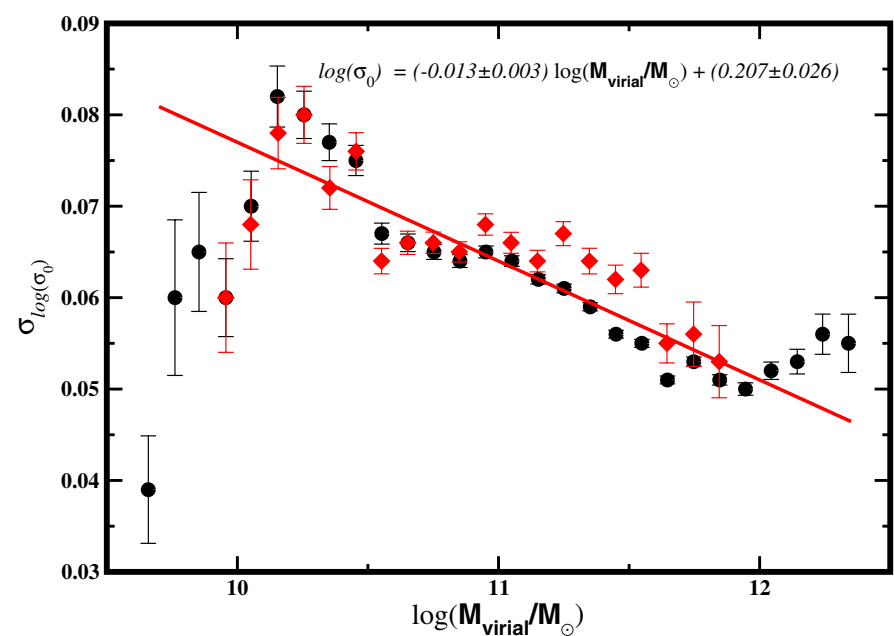

Fig. 6. Variation in the FJR intrinsic dispersion $\left(\sigma_{\log \left(\sigma_{0}\right)}\right)$ in $0.1-\log \left(\mathbf{M}_{\text {virial }} / \mathbf{M}_{\odot}\right)$ wide intervals. Circles represent the total SDSS sample and diamonds the homogeneous SDSS sample. The straight line represents a fit $\left(B C E S_{\text {Bis }}\right)$ to the points in the homogeneous sample for $\log \left(\mathbf{M}_{\text {virial }} / \mathbf{M}_{\odot}\right) \geq 10.5$.

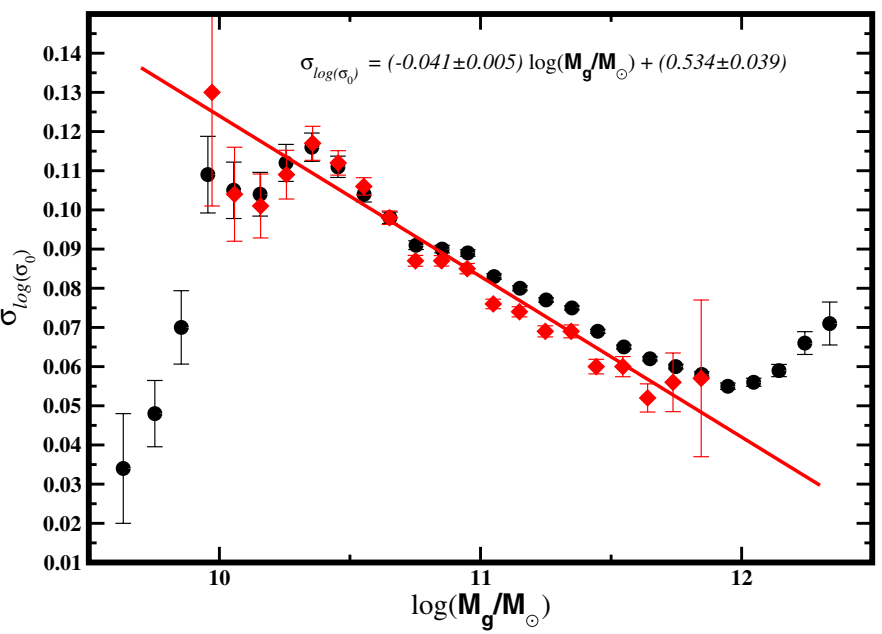

Fig. 7. Variation in the FJR intrinsic dispersion $\left(\sigma_{\log \left(\sigma_{0}\right)}\right)$ in $0.1-\log \left(\mathbf{M}_{\mathbf{g}} / \mathbf{M}_{\odot}\right)$ wide intervals. Circles represent the total SDSS sample and diamonds the homogeneous SDSS sample. The straight line represents a fit $\left(B C E S_{\text {Bis }}\right)$ to the points in the homogeneous sample for $\log \left(\mathbf{M}_{\mathrm{g}} / \mathbf{M}_{\odot}\right) \geq 10.5$. $\mathbf{M}_{\mathrm{g}}$ represents the stellar mass.

we present the values of the FJR intrinsic dispersion as a function of the stellar mass. This figure shows that the behaviour of the intrinsic dispersion as a function of the stellar mass is similar to the behaviour of the intrinsic dispersion as a function of the virial mass, in the sense that, the intrinsic dispersion value changes systematically as we consider more massive galaxies, and that more massive galaxies have a smaller intrinsic dispersion than less massive galaxies. The run test confirms an underlying trend between the stellar mass and the intrinsic dispersion with a confidence level of approximately $99 \%$.

To characterise the behaviour of the intrinsic dispersion as a function of the virial mass, we fitted the points of the homogeneous sample for $\log \left(\mathbf{M}_{\text {virial }} / \mathbf{M}_{\odot}\right) \geq 10.5$ in Fig. 6 to a straight line whose equation is

$\sigma_{\log \left(\sigma_{0}\right)}=(-0.013 \pm 0.003) \log \left(\mathbf{M}_{\text {virial }} / \mathbf{M}_{\odot}\right)+(0.207 \pm 0.026)$ 
As for the case of stellar mass (Fig. 7), we fitted the homogeneous sample points for $\log \left(\mathbf{M}_{\mathrm{g}} / \mathbf{M}_{\odot}\right) \geq 10.5$ to a straight line whose equation is

$\sigma_{\log \left(\sigma_{0}\right)}=(-0.041 \pm 0.005) \log \left(\mathbf{M}_{\mathbf{g}} / \mathbf{M}_{\odot}\right)+(0.534 \pm 0.039)$.

Equation (5) confirms that the correlation between virial mass and the velocity dispersion does not cause the behaviour of the intrinsic dispersion described by Eq. (4), although this correlation might be behind the differences observed between the coefficients of both equations. In the following section, we analyse the possible origin of these differences.

\subsubsection{Differences between virial and stellar mass}

The difference between the coefficients in Eqs. (4) and (5) may be caused by various factors. One possibility is that the virial and stellar masses differ intrinsically (i.e. that the fit slope to both masses differ from 1). Other possible factors are that various processes cause the dispersion in the virial mass as a function of stellar mass to be relatively large (see Fig. 2), and the dispersion in the velocity dispersion as a function of virial mass has a different behaviour from the dispersion in the velocity dispersion as a function of stellar mass (see Figs. 3 and 4). In what follows, we analyse these four possibilities:

- Differences might be caused by the method used to calculate the mass. In accordance with Eq. (3), virial mass consists of both stellar mass and dark matter. In our case, however, we see that the contribution of the dark matter is not very important because the slope of the virial mass versus the stellar mass is approximately 1 (see Fig. 2). So this does not explain the reported difference.

- Differences might be caused by the errors in the parameters used to determine the mass estimates. To explain the dispersion in the stellar mass distribution (see Eq. (2) and Fig. 2), the errors in the magnitude (filter $g$ ) would have to be of the order 1\%; however, the mean errors in this magnitude are approximately of the order $0.1 \%$. For the dispersion in the virial mass (see Eq. (3) and Fig. 2), the error in the velocity dispersion would have to be of the order $10 \%$; however, the mean error in this velocity dispersion is approximately $1 \%$. If the differences were caused by the error in effective radius, this error would have to be of the order $20 \%$, but the mean effective radius error is instead of the order $4 \%$. An error combination in both variables might explain the differences. These errors should be at least of the order $5 \%$ for the velocity dispersion and $10 \%$ for the effective radius. From this, we can say that the errors in the variables involved in the mass estimates are not responsible for the dispersion between the values of the virial and the stellar masses.

- Differences might be caused by the correlation between virial mass, velocity dispersion and effective radius (see Eq. (3)). If there were a dispersion in the effective radius not caused by errors, this dispersion would affect the calculation of the virial mass but not that of the stellar mass, since this mass is independent of the effective radius (see Eq. (2)). This possibility cannot be discarded a priori.

- Differences might be caused by a missing parameter in the calculation of either the virial or the stellar mass or both. The dispersion in the masses might be caused by a missing parameter that is necessary to calculate either the virial or stellar mass or both. This possibility cannot be discarded a priori.

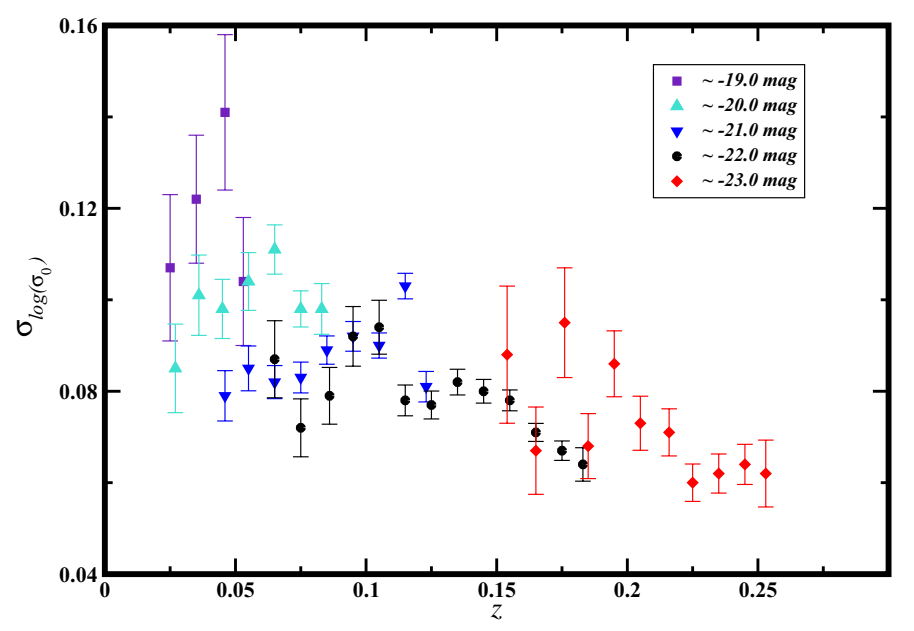

Fig. 8. Intrinsic dispersion in the FJR $\left(\sigma_{\log \left(\sigma_{0}\right)}\right)$ as a function of redshift. Each symbol and colour represent an approximately constant magnitude ( $g$ filter).

As expressed above, cases 3 and 4 could play an important role in the behaviour of the intrinsic dispersion in these masses as a function of the velocity dispersion. Neither of these cases can be discarded a priori, as we only know that the final result of the action that one or the other may cause is different. What is the correct value of the mass? Or which of Eqs. (4) or (5) evaluates the mass the most precisely? The answers to these questions are complicated and, at present, we are unable to provide the correct answer. As a consequence, we use both masses to investigate the universality of the FJR and the universality of the other structural relations.

An interesting point that arises when comparing virial and stellar masses (see 1 above) is that the slope of the fit is approximately equal to 1 . This result is different from those of Cappellari et al. (2006), Robertson et al. (2006), and Koopmans $\&$ Treu (2010), who find that the slope is up to $30 \%$ larger, meaning that within the virial radius up to a $30 \%$ of the total mass is in the form of dark matter. What we find in this paper contradicts this result and shows that the amount of dark matter within the virial radius is negligible. We consider that, given its relevance, this result should be analysed in greater depth, as we propose to do in a future paper.

\subsubsection{The intrinsic dispersion in the FJR as a function of redshift}

An interesting property of the SDSS sample used in this work is that it contains galaxies in the redshift range $0.01<z<0.35$, so that, we can study the behaviour of the structural properties as a function of redshift (see also Shankar et al. 2011). To avoid being affected by the geometrical effect, we can study the behaviour of the intrinsic dispersion in the FJR in either of two ways: (1) studying samples of galaxies of approximately equal magnitude but different redshift, and (2) considering samples of galaxies of approximately equal mass but different redshift.

Figure 8, shows the behaviour of the intrinsic dispersion in the FJR as a function of redshift. Each symbol and colour represent a sample of galaxies of approximately constant magnitude. In some cases, the errors and the narrow redshifts ranges where the samples are distributed make it difficult to observe any trend. However, for $M_{g} \sim-22.0$ and $M_{g} \sim-23.0$ we have data of relatively good quality so we are able to see a smooth trend (see 


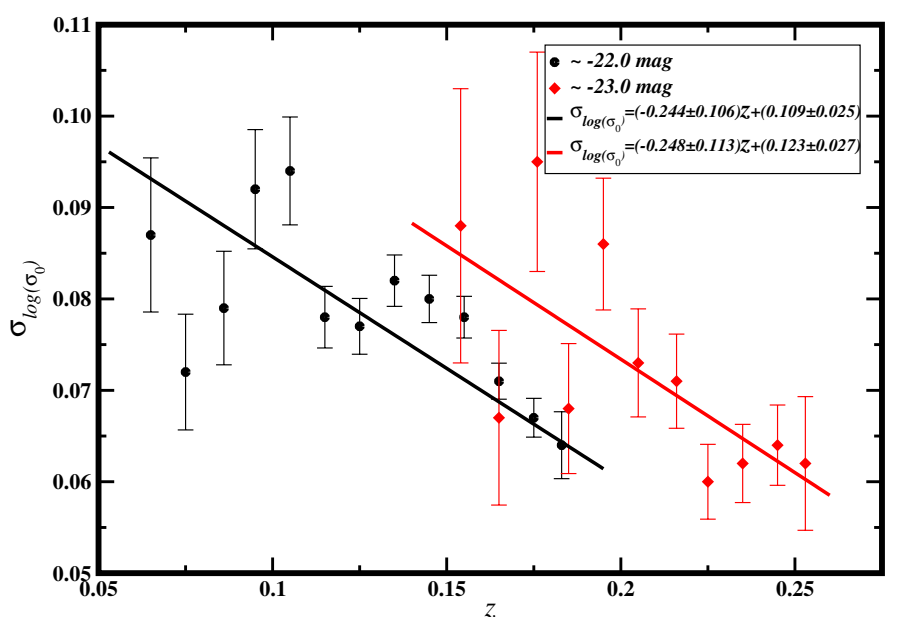

Fig. 9. Intrinsic dispersion in the FJR $\left(\sigma_{\log \left(\sigma_{0}\right)}\right)$ as a function of redshift. Each symbol and colour represent an approximately constant magnitude ( $g$ filter). The straight lines represent fits $\left(B C E S_{\text {Bis }}\right)$ to all the points in each sample.

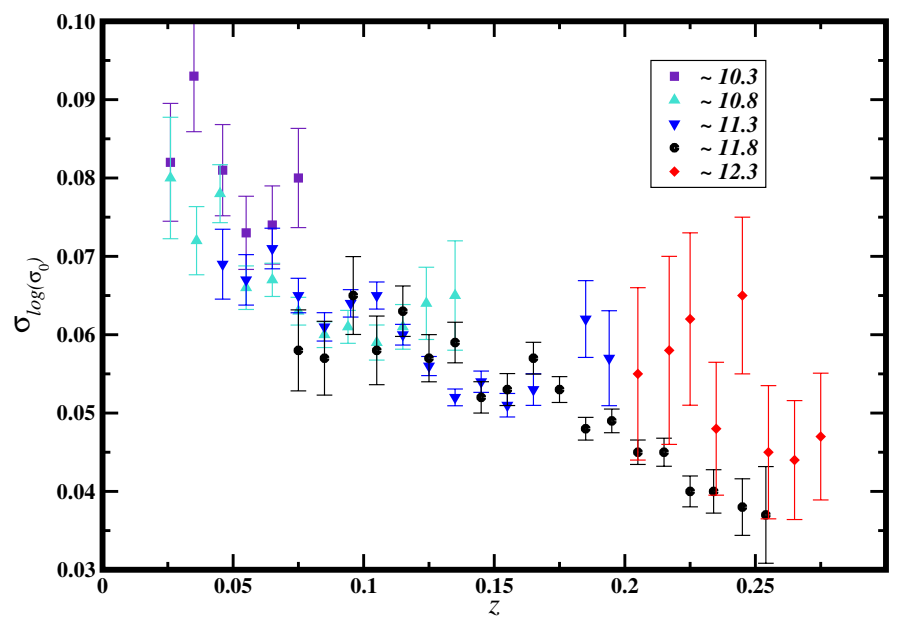

Fig. 10. Intrinsic dispersion in the FJR $\left(\sigma_{\log \left(\sigma_{0}\right)}\right)$ as a function of redshift. Each symbol and colour represent an approximately constant virial mass.

Fig. 9). The run test confirms an underlying trend with a confidence level of approximately $90 \%$.

To characterise the behaviour of the intrinsic dispersion as a function of the redshift, in Fig. 9 we have fitted the points of the sample with $M_{g} \sim-22$ to a straight line whose equation is

$\sigma_{\log \left(\sigma_{0}\right)}=(-0.244 \pm 0.106) z+(0.109 \pm 0.025)$.

Similarly the straight line equation obtained from the sample with $M_{g} \sim-23$ is

$\sigma_{\log \left(\sigma_{0}\right)}=(-0.248 \pm 0.113) z+(0.123 \pm 0.027)$.

For the mass case, in Fig. 10 we can see the behaviour of the intrinsic dispersion in the FJR as a function of redshift. In this graph, each symbol and colour represent a sample of galaxies of approximately constant virial mass. In this case, we find that the errors and the narrow redshifts ranges where some samples are distributed make it difficult to observe any trend. However, for the samples with $\log \left(\mathbf{M}_{\text {virial }} / \mathbf{M}_{\odot}\right) \sim 11.3$ and $\log \left(\mathbf{M}_{\text {virial }} / \mathbf{M}_{\odot}\right) \sim$ 11.8 , the data are of sufficiently good quality for us to discern a clear trend (see Fig. 11). The run test confirms an underlying trend with a confidence level of approximately $95 \%$.

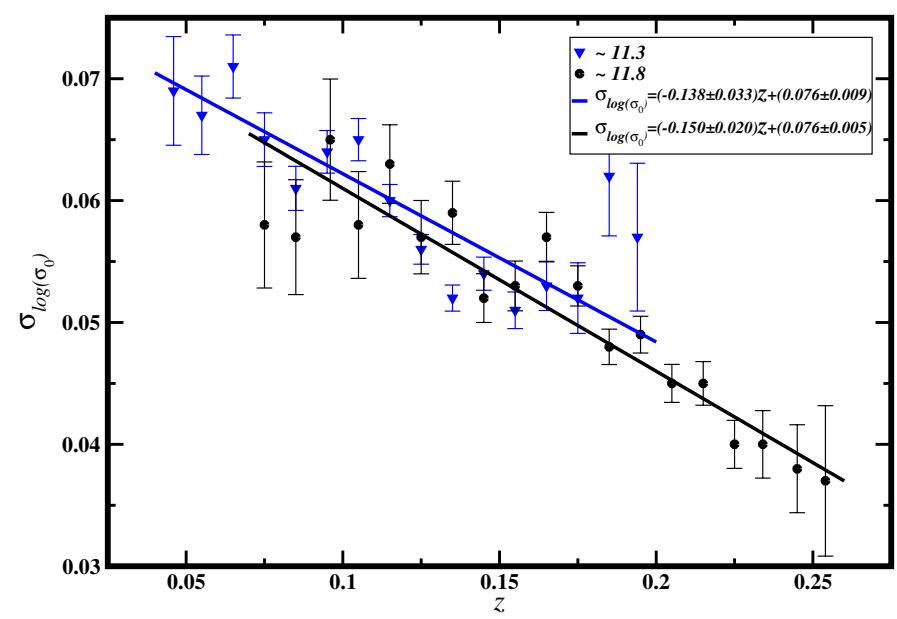

Fig. 11. Intrinsic dispersion in the FJR $\left(\sigma_{\log \left(\sigma_{0}\right)}\right)$ as a function of redshift. Each symbol and colour represent an approximately constant virial mass. The straight lines represent fits $\left(B C E S_{\text {Bis }}\right)$ to all the points in each sample.

To characterise the behaviour of the intrinsic dispersion as a function of redshift, in Fig. 11 we have fitted the points of the sample with $\log \left(\mathbf{M}_{\text {virial }} / \mathbf{M}_{\odot}\right) \sim 11.3$ to a straight line whose equation is

$\sigma_{\log \left(\sigma_{0}\right)}=(-0.138 \pm 0.033) z+(0.076 \pm 0.009)$

Similarly, the straight line equation obtained from the sample with $\log \left(\mathbf{M}_{\text {virial }} / \mathbf{M}_{\odot}\right) \sim 11.8$ is

$\sigma_{\log \left(\sigma_{0}\right)}=(-0.150 \pm 0.020) z+(0.076 \pm 0.005)$.

We must note that the dependence of the intrinsic dispersion in the FJR on redshift has been found for the brightest and more massive galaxies, because for faint, low-mass galaxies we have no data or those available are of insufficiently good quality. On the other hand, the coefficients of Eqs. (6) and (7) are compatible, within the errors, with the coefficients of Eqs. (8) and (9). This behaviour may be understood from Fig. 2, where we show that the difference between the virial and the stellar mass is smaller for larger values of the mass. Given that the objects we use for the study of the intrinsic dispersion as a function of the redshift are massive, the differences that can result should be relatively small.

The results presented in this section, allow us to affirm that, for the brighter and more massive galaxies, the intrinsic dispersion in the FJR changes systematically with distance. Those galaxies located further away, have a lower intrinsic dispersion in the FJR than those located closer by.

\section{Discussion}

The structural relations of ETGs, in particular the FP, have been studied extensively over the past 20-30 years. The commonly presented physical explanation of the FP assumes that the ETGs are in virial equilibrium and are homologous systems. In this context, the term homology means the regular behaviour of both the mass-luminosity ratio and the structure along the entire range of ETG luminosities. However, these assumptions are insufficient to explain the observational results. To explain the differences between theory and observation, which relate in particular to the tilt of the FP, several authors have invoked different mechanisms that affect the ETGs during their formation and 
evolution. One of the most important attempsts to explain the tilt proposes that the ETGs are non-homologous systems, but to date various studies (e.g. Pahre et al. 1998; Scodeggio et al. 1998; Robertson et al. 2006; Jun \& Im 2008) have only been able to explain partially the tilt, and there have been no conclusive results.

Nigoche-Netro et al. (2009) demonstrated that the FP is not a simple plane in the space of parameters $\log \left(r_{\mathrm{e}}\right),\langle\mu\rangle_{\mathrm{e}}$ and $\log \left(\sigma_{0}\right)$ and that the distribution of galaxies in this space depends on luminosity. In the case of the KR and FJR, NigocheNetro et al. $(2008,2010)$ found the same behaviour. Fraix-Burnet et al. (2010) discovered that the distribution of galaxies in the parameter space considered is very complex, and established that the FP and other structural relations such as the FJR are formed by seven different groups of galaxies. These groups occupy different separate regions on the graphical planes or volumes for which the structural relations are plotted. They also find that each group is truly "homologous", where "homology", for them, means similarity caused by their having the same class of progenitor. Each group follows its own structural relation, which is more loosely defined for less-diversified groups. In this context, "diversity" depends on the number and nature of transformation events that affect the galaxies during their life time such as: collapse, accretion, interaction, and merging. Hence the term lessdiversified (more-diversified) means that galaxies have suffered fewer (more) transformation events during their life times.

It is important not to confuse the terms "diversity" and "diversification". "Diversification", following Fraix-Burnet et al. (2006), refers to the number of different classes of objects that are present in a sample. An illustrative example of the difference between these two concepts is the following. Four identical galaxies when mixed in pairs would produce two different galaxies, which would be different among themselves and differ from the original galaxies. If these two galaxies were mixed again, the mixture would produce yet another class of galaxy. In this example, during the entire process, four different classes of objects have been produced, but the final result is that there is only one class of galaxy and this last object is the more diversified, because it has suffered the largest number of transformation events.

Fraix-Burnet et al. (2010) concluded that the FP might be just an historical and not a physical correlation, so that, the correlation among $\log \left(r_{\mathrm{e}}\right),\langle\mu\rangle_{\mathrm{e}}$, and $\log \left(\sigma_{0}\right)$ might not be a tilted virial plane because of dissipation or a particular behaviour of $\mathbf{M} / L$, but rather a parametric correlation between the evolution of these parameters. In other words, the FP would be the result of several transforming events such as collapse, accretion, interaction, and merging, that is to say, the FP would be the result of the historical sequence of events that affected, one way or another, the physical structure of the galaxies in question.

In previous sections, we have characterised the variation in the distribution of the ETGs in the plane of the parameters $M$ and $\log \left(\sigma_{0}\right)$ using the intrinsic dispersion. We have found that the intrinsic dispersion in the FJR depends on the luminosity and mass of the galaxies and that the distribution of brighter and more massive galaxies has a lower intrinsic dispersion than that for the fainter and less massive galaxies. This result is in agreement with the work of Fraix-Burnet et al. (2010), where, in their Fig. 7 we can see that the region of the brighter galaxies on the FJR plane is formed by the more diversified groups - only one or two groups populate this region -, while the region of the fainter galaxies is formed by the less diversified groups - four or five groups populate this region -, which occupy a more extended region. Put differently, the distribution of galaxies inside the groups and the distribution of the groups on the FJR plane might be responsible for producing the distribution of brighter galaxies, which appear to be those that are more diversified, and have a lower intrinsic dispersion than the distribution of fainter galaxies, which seem to be the less diversified ones. Hence, we may conclude that the intrinsic dispersion in the FJR might depend on several transforming events such as collapse, accretion, interaction, and merging, in other words, just as for the FP, the intrinsic dispersion in the FJR might be a consequence of the historical series of events that affected the physical structure of the galaxies.

We have also found that, for bright and massive galaxies in the redshift range $0.01<z<0.25$, the intrinsic dispersion in the FJR depends on redshift and that the distribution of the more distant galaxies has a smaller intrinsic dispersion than more nearby galaxies. Fraix-Burnet et al. (2010) found no strong relation between their groups and redshift, although, the redshift range that they studied is very narrow $(0.007<z<0.053)$, such that the dependence of groups on redshift might have gone unnoticed.

The results that we have mentioned above are preliminary, given that the sample used in this paper and that of Fraix-Burnet et al. (2010) are different. The former sample corresponds to ETGs with different environments and redshifts, while the latter corresponds to ETGs in nearby clusters. Prior to taking these results as conclusive, it is necessary to perform an analysis of the diversification of the galaxies for samples in different environments and at different redshifts. It is also important to note that the behaviour of the intrinsic dispersion, which we have found, might be due to the involvement of other phenomena. This may include phenomena that Fraix-Burnet et al. (2010) did not contemplate, such as that the dispersion might be partially caused by the objects from which they originated, and not only the transformation events that they have undergone during their lifetimes.

\section{Conclusions}

Analysing the intrinsic dispersion in the FJR for a sample of approximately 90000 ETGs from the literature, we have found the following:

- The intrinsic dispersion of FJR depends on luminosity, mass, and redshift.

- The distribution of brighter and more massive galaxies has a smaller intrinsic dispersion than the distribution for the fainter and less massive galaxies.

- In the redshift range $0.01<z<0.25$, for luminous and massive galaxies, the distribution of the more distant galaxies has a smaller intrinsic dispersion than that for the more nearby galaxies.

Fraix-Burnet et al. (2010) showed that the structural relations, among which they include the FJR, are formed by seven different groups of galaxies. These groups define separate regions in the structural relations. Each group follows its own structural relation, which is more loosely defined for less-diversified groups. In this context, "diversity" means the number and the nature of transformation events that affect the galaxies during their life times. When we compared our results with those of Fraix-Burnet et al. (2010), we found that the distribution of galaxies inside the groups and the distribution of the groups on the FJR plane might cause the brighter (more-diversified) galaxies to have a smaller intrinsic dispersion than the fainter (less-diversified) galaxies. Hence, we may conclude that the intrinsic dispersion in the FJR might depend on the number and the nature of transformation 
events that affect the galaxies such as collapse, accretion, interaction, and merging, that is to say, the intrinsic dispersion might depend on the evolutionary histories of the galaxies.

As mentioned above (Sect. 3), before taking the former results as conclusive, further diversification studies should be carried out in ETG samples similar to the one presented in this paper. It is also important to consider that the intrinsic dispersion might depend on other factors that have not been considered in the analysis by Fraix-Burnet et al. (2010), such as that part of this dispersion might be due to the original inherent properties of the galaxies and not only to the transformation events that they have experienced during their life times.

Finally, as seen in Sect. 2.3, a comparison of virial and stellar mass returns a value of the slope approximately equal to 1 , a value that differs from recent results in the literature in which the slope could be as large as $\sim 1.3$. This would mean that within the virial radius $30 \%$ of the mass could be in the form of dark matter. Our results indicate that within this radius the amount of dark matter is negligible. We shall discuss this point in a forthcoming paper.

Acknowledgements. We would like to dedicate this humble work to the memory of Mrs. Eutiquia Netro Castillo, an extraordinary woman.

We would like to thank Consejo Nacional de Ciencia y Tecnología (México) for a postdoctoral fellowship number 117993, Instituto de Astrofísica de Andalucía (IAA, España), Instituto de Astrofísica de Canarias (IAC, España) and Instituto de Astronomía (UNAM, México) for all the facilities provided for the realisation of this project. We acknowledge financial support from projects: CSD2006 00070 "1st Science with GTC", of the CONSOLIDER 2010 Programme; and AYA2010-21887-C04, AYA2007-67965-C03, "Estallidos", PNAYA of the Spanish MICINN. We would also like to thank Jana Benda for her help with this paper.

\section{References}

Abazajian, K. N., Adelman-McCarthy, J. K., Agüeros, M. A., et al. 2009, ApJS, 182,543

Aguerri, J. A. L., \& González-García, A. C. 2009, A\&A, 494, 891

Aguerri, J. A. L., Iglesias-Paramo, J., Vilchez, J. M., \& Muñoz-Tuñón, C. 2004, AJ, 127, 1344

Akritas, M. G., \& Bershady, M. A. 1996, ApJ, 470, 706

Barger, A. J., Aragón-Salamanca, A., Smail, I., et al. 1998, ApJ, 501, 522

Barr, J., Jørgensen, I., Chiboucas, K., Davies, R., \& Bergmann, M. 2006, AJ, 649, L1

Bell, E. F., McIntosh, D. H., Katz N., \& Weinberg M. D. 2003, ApJS, 149, 289

Bender, R., Burstein, D., \& Faber S. M. 1992, ApJ, 399, 462

Bender, R., Saglia, R. P., Ziegler, B., et al. 1998, ApJ, 493, 529

Bernardi, M., Sheth, R. K., Annis, J., et al. 2003a, AJ, 125, 1817

Bernardi, M., Sheth, R. K., Annis, J., et al. 2003b, AJ, 125, 1849

Bernardi, M., Sheth, R. K., Annis, J., et al. 2003c, AJ, 125, 1866

Blakeslee, J. P., Lucey, J. R., Tonry, J. L., et al. 2002, MNRAS, 330, 443

Caon, N., Capaccioli, M., \& D'Onofrio, M. 1993, MNRAS, 265, 1013

Cappellari, M., Bacon, R., Bureau, R., et al. 2006, MNRAS, 366, 1126

de la Rosa, I. G., de Carvalho R. R., \& Zepf, S. E. 2001, AJ, 122, 93
Denicoló, G., Terlevich, R., Terlevich, E., et al. 2005, MNRAS, 356, 1440

Desroches, L. B., Quataert, E., Ma, C. P., \& West A. A. 2007, MNRAS, 377, 402 Djorgovsky, S., \& Davies, M. 1987, ApJ, 313, 59

Dressler, A., Lynden-Bell, D., Burstein, D., et al. 1987, ApJ, 313, 42

Evstigneeva, E. A., Reshetnikov, V. P., \& Sotnikova, N. Y. 2002, A\&A, 381, 6

Faber, S. M., \& Jackson, R. E. 1976, ApJ, 204, 668

Fraix-Burnet, D., Douzery, E. J. P., Choler, P., \& Verhamme, A. 2006, J. Class, 23, 57

Fraix-Burnet, D., Dugué, M., Chattopadhyay, T., Chattopadhyay, A. K., \& Davoust, E. 2010, MNRAS, 407, 2207

Fritz, A., Böhm, A., \& Ziegler, B. L. 2009, MNRAS, 393, 1467

Gonzáles-García, A. C., \& van Albada, T. S. 2003, MNRAS, 342, L36

Gutiérrez, C. M., Trujillo, I., Aguerri, J. A. L., Graham, A. W., \& Caon, N. 2004, ApJ, 602, 664

Hamabe, M., \& Kormendy, J. 1987, in Structure and Dynamics of Elliptical Galaxies, ed. T. de Zeeuw (Dordrecht: Reidel), IAU Symp., 127, 379

Hudson, M. J., Lucey, J. R., Smith, R. J., \& Steel, J. 1997, MNRAS, 291, 488

Hyde, J. B., \& Bernardi, M. 2009, MNRAS, 394, 1978

Isobe, T., Feigelson, E. D., Akritas, M. G., \& Babu, G. J. 1990, ApJ, 364, 104

Jørgensen, I., Franx, M., \& Kærgaard, P. 1996, MNRAS, 280, 167

Jørgensen, I., Franx, M., Hjorth, J., \& van Dokkum, P. G. 1999, MNRAS, 308, 833

Jørgensen, I., Bergmann, M., Davies, R., et al. 2005, AJ, 129, 1249

Jørgensen, I., Chiboucas, K., Flint, K., et al. 2006, ApJ, 639, L9

Jun, H. D., \& Im, M. 2008, ApJ, 678, 97

Kelson, D. D., van Dokkum, P. G., Franx, M., Illingworth, G. D., \& Fabricant, D. 1997, ApJ, 478, L13

Kjærgaard, P., Jørgensen, I., \& Moles, M. 1993, ApJ, 418, 617

Koopmans, L. V. E., \& Treu, T. 2010, HiA, 15, 61

Kormendy, J. 1977, ApJ, 218, 333

Kormendy, J. 1985, ApJ, 295, 73

La Barbera, F., Busarello, G., Merluzzi, P., Massarotti, M., \& Capaccioli M. 2003, ApJ, 595, 127

La Barbera, F., Govone, G., Busarello, G., et al. 2005, MNRAS, 358, 1116

La Barbera, F., Busarello, G., Merluzzi, P., et al. 2008, ApJ, 689, 913

Nigoche-Netro, A. 2007, Ph.D. Thesis. Universidad Complutense de Madrid

Nigoche-Netro, A., Moles, M., Ruelas-Mayorga, A., Franco-Balderas, A., \& Kjærgaard, P. 2007, A\&A, 472, 773

Nigoche-Netro, A., Ruelas-Mayorga, A., \& Franco-Balderas, A. 2008, A\&A, 491, 731

Nigoche-Netro, A., Ruelas-Mayorga, A., \& Franco-Balderas, A. 2009, MNRAS, 392,1060

Nigoche-Netro, A., Aguerri, J. A. L., Lagos, P., et al. 2010, A\&A, 516, A96

Pahre, M. A., de Carvalho, R. R., \& Djorgovsky, S. G. 1998, AJ, 116, 1606

Reda, F. M., Forbes, D. A., Beasley, M., O’Sullivan, E. J., \& Goudfrooij, P. 2004, MNRAS, 354, 851

Reda, F. M., Forbes, D., \& Hau, G. 2005, MNRAS, 360, 693

Robertson, B., Cox, T. J., Hernquist, L., et al. 2006, ApJ, 641, 21

Scodeggio, M., Gavazzi, G., Belsole, E., Pierini, D., \& Boselli, A. 1998, MNRAS, 301, 1001

Shankar, F., Marulli, F., Bernardi, M., et al. 2011, MNRAS, submitted [arXiv: $1105.6043 \mathrm{~S}]$

Treu, T., Stiavelli, M., Bertin, G., Casertano, S., \& Møller P. 2001, MNRAS, 326,237

Treu, T., Ellis, R. S., Liao, T. X., et al. 2005, ApJ, 633, 174

Trujillo, I., Aguerri, J. A. L., Gutiérrez, C. M., \& Cepa, J. 2001, AJ, 122, 38

Trujillo, I., Aguerri, J. A. L., Gutiérrez, C. M., Caon, N., \& Cepa, J. 2002, ApJ, 573,9

York, D. G., Adelman, J., Anderson, J. E., Jr., et al. 2000, AJ, 120, 1579

Ziegler, B. L., Saglia, R. P., Bender, R., et al. 1999, A\&A, 346, 13 